FINANÇAS 


\title{
IMPACTOS DA ZELOTES NAS AÇÕES DAS EMPRESAS ENVOLVIDAS
}

\author{
IMPACTS OF THE ZELOTES IN THE SHARES \\ OF THE INVOLVED COMPANIES
}

Paulo Schmidt

Universidade Federal do Rio Grande do Sul

Marco Antonio dos Santos Martins

Universidade Federal do Rio Grande do Sul

José Luiz dos Santos

Faculdade São Francisco de Assis
Gilberto de Oliveria Kloeckner

Faculdade São Francisco de Assis
Data de submissão: | | ago. 20 |7. Data de aprovação:

20 jan. 20।8. Sistema de avaliação: Double blind review. Universidade FUMEC / FACE. Prof. Dr. Henrique Cordeiro Martins. Prof. Dr. Cid Gonçalves Filho.

\section{RESUMO}

O objetivo deste estudo é apresentar quais os impactos da divulgação dos eventos da Zelotes nas cotações das ações das empresas brasileiras listadas na Bolsa de Valores de São Paulo - BOVESPA citadas nessa operação. A análise foi realizada utilizando o modelo de Estudo de Eventos. Foram analisados os impactos em três empresas abertas brasileiras. $O$ estudo indica que os resultados apurados não são significantes, com $95 \%$ de confiança, validando, portanto, a hipótese nula, ou seja, não há diferenças significativas entre o retorno normal e o retorno anormal. Portanto, foi possível concluir, preliminarmente, que o mercado foi indiferente, no longo prazo, às denúncias envolvendo as empesas analisadas, corroborando a tese de que o mercado brasileiro não reage de maneira significativa aos problemas de corrupção.

\section{PALAVRAS-CHAVE}

Operação Zelotes. Estudo de Eventos. Corrupção. Empresas Brasileiras. Capital Aberto. 


\section{ABSTRACT}

The objective of this study is to show the impacts of the disclosure of Zelotes events on the stock prices of Brazilian companies cited in this operation and listed on the São Paulo Stock Exchange.The analysis was performed using the Event Study method. Impacts on three Brazilian public companies were analyzed. The study suggests that the results are not significant with $95 \%$ confidence, thus not allowing the rejection of the null hypothesis, that is, there were no significant differences between normal and abnormal returns. It was preliminarily possible to conclude that the market seems to be indifferent in the long run to complaints involving the studied companies, therefore supporting the thesis that the Brazilian market does not significantly react to corruption issues.

\section{KEYWORDS}

\section{Zelotes Operation. Event Study. Corruption. Brazilian Companies. Open Capital.}

\section{INTRODUÇÃO}

O Brasil passa por um momento de instabilidade política com o aumento da desconfiança internacional sobre a sua habilidade de superá-la, gerando um quadro de forte retração econômica, com inflação, desemprego, volatilidade cambial, altas taxas de juros e aumento da economia informal, pois a elevação do nível de desemprego obriga os desempregados a buscarem alternativas à margem da formalidade, levando ainda a desestruturação de muitos setores produtivos, a precariedade do trabalho e desigualdade da renda (MATTOSO, 20 l6; BALTAR, 20 I 6; CACCIAMALI, 20 I6).

Muito dessa crise pode ser atribuída a cultura corruptiva instituída no Brasil, gerada pela continuada corrupção de gestores públicos e políticos, resultando numa crescente alienação do povo em relação à política e o aumento da percepção de que as autoridades públicas não são confiáveis (BAQUERO, 20I5). Para Queiroz (20I5), o impacto da corrupção no Brasil é tão sig- nificativo que pode comprometer mais de 2,5\% do Produto Interno Bruto (PIB) do país, chegando a US\$50 bilhões/ano.

O crescente aumento da corrupção e de improbidades administrativas cometidas nas licitações públicas nos últimos anos no Brasil, tem despertado a atenção de juristas, doutrinadores, da Polícia Federal (PF) e do Ministério Público Federal (MPF) (NASCIMENTO e BARACHO, 2016).

Nos últimos anos, foram realizadas inúmeras operações da PF e MPF, com destaque para as operações: Operações Lava Jato, Acrônico, Caixa de Pandora, Castelo de Areia, Mensalão, Satiagraha, Greenfield e Zelotes. Essa última, iniciou em 26 de março de 2015 e foi dedicada à apuração de suspeitas de manipulação, entre 2005 e 2013, em julgamentos de processos de 70 empresas no Conselho Administrativo de Recursos Fiscais (Carf), órgão ligado ao Ministério da Fazenda, totalizando R \$ 19,6 bilhões que deixaram de ser recolhidos aos cofres públicos (MPF, 20I6; SAAD, 20I6). 
Entre as 70 empresas que estão sendo investigadas pela Zelotes, algumas são de capital aberto e negociadas na BOVESPA. Dentre essas empresas com valores investigados acima de $\mathrm{R} \$ 1,0$ bilhão estão o Banco Santander (fraude estimada de R\$ 3,34 bilhões), o Banco Bradesco (fraude estimada de R\$ 2,75 bilhões) e a Gerdau (fraude estimada de R\$ I,22 bilhão). Além dessas três, foram aventadas outras empresas de capital aberto com ações listadas na BOVESPA, porém não houveram citações formais, nem indiciamento na Zelotes, como o caso da Marcopolo, Embraer, BRF, Brascan, BTG e Petrobras. (FOLHA DE SÃO PAULO, 20 I 6; EXAME, 20 I6).

Vários estudos apontam para uma possível relação entre a divulgação de eventos e o preço de mercado das ações dessas empresas a partir dessas externalidades, como os de Fama (1969), Terra e Lima (2006), Gonçalves et al. (20l3), Paulo, Sarlo Neto e Santos (20l3), Wright (20I2), Medeiros e Matsumoto (2006), Grullon, Michenaud e Weston (2015), Batisletta (2004), Peress (20l4), Ramnath (2002), Cohen e Wang (20I3), Arthur (2003), Mitchell e Stafford (2000), Ikenberry e Cavalcante (2009) e de Lucchesi e Famá (2007).

Diante desse atual cenário, percebe-se a importância de pesquisar quais os impactos que a divulgação de eventos da Zelotes causa na precificação das empresas citadas nessas operações contra a corrupção.

Portanto, essa investigação objetiva apresentar quais os impactos da divulgação dos eventos relacionados com a Zelotes nas cotações das ações das empresas brasileiras de capital aberto listadas na BOVESPA citadas nessa operação.

Para análise desses impactos, será utilizado o modelo de Estudo de Eventos. Para
Bergmann et al. (20I5, p.I I08), “o modelo de estudo de evento busca compreender e analisar o impacto que um evento específico gera em uma variável qualquer”.

Esse estudo apresenta, além da introdução, uma seção dedicada à fundamentação teórica sobre teoria de eventos, uma sobre a Zelotes, uma sobre procedimentos metodológicos, uma seção que apresenta os resultados encontrados e uma com as considerações finais e indicações de estudos a serem realizados no futuro.

\section{ESTUDOS DE EVENTOS}

O estudo de eventos busca demonstrar o resultado de um evento sobre a precificação das ações de uma empresa e a reação do mercado ao evento e seu impacto sobre o preço da ação. Em síntese, o método busca identificar até que ponto o preço das ações está sendo impactado por um determinado evento. Os eventos analisados podem ser gerados pela própria empresa, como por exemplo: uma combinação de negócios, a apuração ou distribuição de lucros; ou gerados de agentes externos, como: a criação de uma legislação impactante para a empresa, a movimentação da concorrência ou a associação da empresa a problemas, como é o caso da relação com operações como a Zelotes.

O desenvolvimento da teoria do estudo de eventos foi iniciada por Dolley em 1933, sendo consolidada em estudos subsequentes desenvolvidos por Myers e Bakay (1948), Baker, (1956, 1957, 1958), Ashley (1962), Ball e Brown (1968), Fama, Fisher, Jensen e Roll (1969), Campbell, Lo e Mackinlay (1977) e Brown e Warner (1980, 1985).

Segundo Campbell, Lo e Mackinlay (1977), a consolidação do estudo de eventos se deu com uma investigação dos impactos do des- 
membramento das ações de uma empresa nos preços nominais de suas ações.

Para a aplicação do estudo de eventos, descritos por Campbell, Lo e Mackinlay (1977), foram definidos sete procedimentos:

I. Definição do evento: é necessário definir a data do evento a ser estudado e a janela do evento, identificando - período que será analisado antes (janela de estimação) e depois do evento (janela após o evento), para verificar se houve impacto do evento no preço das ações. A análise de uma janela com período anterior à data zero objetiva à identificação de indícios do uso de informações privilegiadas, enquanto a do período posterior visa fornecer evidências da velocidade e precisão do ajuste dos preços à nova informação liberada ao mercado (CAMARGOS e BARBOSA, 2003);

2. Seleção da amostra: determinação do critério de seleção das empresas a serem incluídas no estudo;

3. Definição dos retornos normal e anormal (modelo de medição): o retorno anormal é o retorno real ex-post do valor da ação, menos o retorno normal durante o período de tempo estudado. Por outro lado, o retorno normal é aquele que seria esperado caso o evento não ocorresse. A apresentação do retorno anormal pode ser visualizada na seguinte equação: $A R_{i, t}=R_{i, t}-E\left[R_{i t} \mid X\right]$,

onde:AR: retorno anormal;

$\mathrm{R}$ : retorno real;

$E[R]$ : retorno normal;

4. Procedimento de estimativa (Seleção do modelo de performance normal): após a definição do retorno anormal, deverá ser realizada a seleção do mo- delo de performance normal, estimando os parâmetros do modelo através de um subconjunto de dados, dentro de uma janela de estimação para testar o procedimento. O período do evento não deve ser incluído no período de estimação para prevenir influências do evento no modelo de performance normal;

5. Procedimento de teste: deve ser calculado o retorno anormal para cada empresa analisada, com o objetivo de verificar se o retorno anormal é diferente do normal, através da determinação da técnica que deverá agregar os retornos anormais das empresas individuais, seguido da definição da hipótese nula, normalmente $\mathrm{AR}_{\mathrm{i}, \mathrm{t}}=0 \mathrm{e}$ da condução dos testes estatísticos.

6. Resultados empíricos: Após as análises estatísticas e de teste da hipótese, onde deve ser verificado se o retorno anormal é diferente do normal, os resultados empíricos devem ser apresentados;

7. Interpretações e conclusões: a última etapa da aplicação do modelo, apresentado por Campbell, Lo e Mackinlay (1977), é a fase de interpretações e conclusões, onde, de forma conjunta com o diagnóstico dos resultados, deve ser apresentado um diagnóstico sobre os resultados alcançados e principais conclusões, que permita levar a conclusões que gerem uma compreensão clara dos mecanismos pela qual os eventos afetaram, ou não, os preços das ações, devendo incluir a distinção em relação a explicações concorrentes.

Essas etapas servirão de fundamento para identificação das anormalidades, caso 
existam, nos retornos das ações das empresas com negociação regular na BOVESPA, citadas na Zelotes.

\section{Cálculo do Retorno Normal}

Para o cálculo do retorno normal existem várias abordagens, que podem ser divididas em: modelos estatísticos e modelos econômicos (CAMPBELL, LO e MACKINLAY, I 997; KAN, ROBOTTI, e SHANKEN, 20I3; PEREIRA, SECURATO, SOUSA, 20I6; DEMIGUEL, NOGALES, UPPAL, 20I4; JUNIOR e CARVALHO, 20I5; PESSANHA et al., 20I4).

\section{Modelos estatísticos}

Os modelos estatísticos estão fundamentados em suposições de que o comportamento do retorno dos ativos não depende de argumentos econômicos. Eles pressupõem que o retorno dos ativos são conjuntamente normais e multivariados e distribuídos identicamente e independentemente ao longo do tempo, divididos em modelo de retorno médio constante, modelo de mercado e outros modelos estatísticos.

O modelo de retorno médio constante, embora seja o mais simples, frequentemente fornece resultados similares a modelos mais sofisticados (BROWN e WARNER, 1980, 1985). Esse modelo pode ser configurado a partir da seguinte equação:

$\mathrm{R}_{\mathrm{it}}=+\sum_{\mathrm{it},} \mathrm{E}\left[\sum_{\mathrm{it}}\right]=0 ; \operatorname{Var}\left[\sum_{\mathrm{it}}\right]=\Upsilon^{2}$.

Onde: $\mathrm{R}_{\mathrm{i}, \mathrm{t}}$ :retorno da ação i no período $\mathrm{t}$;

: retorno médio do ativo

$\mathrm{i} ; \sum_{\mathrm{it}}$ : termo de distúrbio.

Para Polinsky e Shavell (2007), o modelo de mercado relaciona o retorno de uma ação qualquer ao retorno do portfolio de mercado. A especificação linear do modelo supõe uma normalidade comum de retornos dos ativos. Esse modelo foi idealizado para qualquer ação e pode ser representado pela seguinte equação:

$\mathrm{R}_{\mathrm{it}}=\mathrm{a}_{\mathrm{i}}+\mathrm{b}_{\mathrm{i}} \mathrm{R}_{\mathrm{mt}}+\sum_{\mathrm{it}} ; \mathrm{E}\left[\sum_{\mathrm{it}}\right]=0 \operatorname{Var}\left[\sum_{\mathrm{it}}\right]=\int^{2}$.

Onde: $R_{i, t}$ : retorno da ação i no período $t$;

$a_{i} b_{i}$ : parâmetros do modelo de mercado;

$\mathrm{R}_{m, \mathrm{t}}^{\mathrm{t}}$ : retorno do portfólio de mercado; $\sum_{i t}$ : termo de distúrbio.

Para Campbell, Lo e Mackinlay (1997), os outros modelos estatísticos propostos para modelar o retorno normal, como o de fatores, possui o benefício de reduzir a variância do retorno anormal através de uma maior explicação do retorno normal, que pode ser dividido em modelo de um fator (modelo de mercado) e modelo de multifatores, como os modelos de índices da indústria Sharpe (1970) e Sharpe, Alexander e Bailey (1995).

\section{Modelos econômicos}

Os modelos econômicos para o cálculo do retorno normal buscam restringir os parâmetros dos modelos estatísticos para fornecer um modelo mais contraído de retorno normal. Os modelos econômicos mais comuns são o CAPM (Capital Asset Pricing Model) e o APT (Arbitrage Pricing Theory) (ZABARANKIN e URYASEV, 20I4; ELBANNAN, 20I5).

O CAPM foi apresentado nos estudos de Sharpe (1964) e Lintner (1965), apresentando uma teoria de equilíbrio onde o retorno esperado de um dado ativo é uma função linear de sua covariância com o retorno do portfolio de mercado.

O APT, desenvolvido por Ross (1976), apresenta uma teoria de precificação de ativos onde, na ausência de uma arbitragem assintótica, o retorno esperado de um dado ativo é determinado pela sua covariância com fatores múltiplos. 


\section{Estudos Relacionados}

Vários estudos de eventos ligados à análise de variáveis do mercado de capitais brasileiro já foram realizados. Sendo que podem ser elencados alguns como os de Batistella et al. (2004), Medeiros e Matsumoto (2006), Terra e Lima (2006) e Camargos e Barbosa (2003).

Batistella et al. (2004) investigaram que o evento da adoção de algum dos níveis de governança corporativa do Novo Mercado da BOVESPA, provavelmente, não interferiram no retorno das ações das companhias investigadas.

Medeiros e Matsumoto (2006) analisaram como o evento de emissões públicas de ações de empresas brasileiras na BOVESPA impactaram no retorno dessas ações. Os principais resultados encontrados indicam que houveram retornos anormais negativos na data do anúncio do evento e que no período de uma ano após os eventos as ações obtiveram retornos negativos, considerando os ajustes ao risco e ao mercado.

Terra e Lima (2006) pesquisaram, com o uso do estudo de eventos, como a divulgação das demonstrações contábeis anuais e trimestrais das empresas que negociam ações na BOVESPA, afetam igualmente a todas empresas ou se existe alguma diferenciação para as que adotaram boas práticas de governança corporativa. Os principais resultados indicam que o mercado reage de forma diferenciada quando as empresas sinalizam que adotaram boas práticas de governança corporativa.

Camargos e Barbosa (2003) verificaram, como o uso de estudo de eventos, se o mercado de capitais brasileiro pós-Plano Real passou a demonstrar eficiência informacional semiforte com o anúncio de fusões e aquisições de empresas com ações na BOVESPA. O resultado apurado na pesquisa foi de que, pelo aspecto informacional, o mercado de capitais brasileiro não se comportou de maneira eficiente, considerando à forma semiforte.

\section{EVENTOS DA OPERAÇÃO ZELOTES}

A operação Zelotes apresentou, desde a sua deflagração em 26/03/20I5, sete fases, envolvendo a PF e o MPF. A cronologia completa da operação foi apresentada pela Folha de São Paulo (2016).

Os eventos em que empresas brasileiras de capital aberto foram citadas e que fazem parte desse estudo, serão apresentados, objetivando identificar os impactos desses eventos no valor das ações dessas empresas.

A Zelotes foi originada de uma carta anônima entregue à PF. Na primeira fase, iniciada em 26/03/20I5, foram cumpridos 41 mandados de busca e apreensão em Brasília, São Paulo e Ceará.

Paralelamente à Zelotes, o Senado Federal instalou uma Comissão Parlamentar de Inquérito (CPI) para investigar o esquema e, em 23/06/20I5, foram convocadas 13 pessoas, entre eles um executivo do Banco Santander.

Da I ${ }^{\mathrm{a}}$ a $5^{\mathrm{a}}$ fases da Zelotes, nenhuma das empresas deste estudo foram citadas.

$\mathrm{Na}$ deflagração da sexta fase, em 25/02/2016, a Gerdau passou a ser investigada por uma suposta compra de decisões no Carf, que somaram $R \$ 1,22$ bilhão. Foram cumpridos 9 mandados de condução coercitiva, sendo um deles do presidente da Gerdau. Em 06/05/2016, ainda nessa fase da operação, o MPF denunciou o Banco Santander como participante do esquema, com uma fraude estimada de $R \$ 3,34$ bilhões.

A sétima fase foi deflagrada em 
09/05/20I6. No dia 16/05/20I6, a PF indiciou o presidente da Gerdau pelos crimes de corrupção ativa, corrupção passiva, lavagem de dinheiro, tráfico de influência, entre outros. Em 3 I/05/20 I 6, A PF indiciou o presidente do Bradesco como participante do esquema de compra de decisões no Carf, com fraude estimada de $\mathrm{R} \$ 2,75$ bilhões.

As citações da Gerdau e do Bradesco na Zelotes, desencadearam novas ações fora do país. Em 26/05/2016, foi protocolada em um tribunal de Nova York uma ação coletiva de investidores que compraram ações da Gerdau entre 02/06/20 I I e I5/05/20 I6. Em 03/06/2016, foi aberto um processo contra - Bradesco em um tribunal de Manhattan em nome de investidores que adquiriram American Depositary Receipt (ADRs) do Bradesco entre 30/04/20I2 e 31/05/2016.

\section{PROCEDIMENTOS METODOLÓGICOS}

As empresas selecionadas para integrar esse estudo quantitativo foram as S.As. listadas na BOVESPA, citadas formalmente na Zelotes, com negociação no período de vigência da operação e com suspeita de fraude em julgamentos do Carf. Nessas condições, foram identificadas o Santander, a Gerdau e - Bradesco. As outras empresas de capital aberto que foram apenas aventadas na operação, porém, não citadas formalmente, nem indiciadas na Zelotes, foram a Marcopolo, Embraer, BRF, Brascan, BTG e Petrobras, não fazem parte do estudo.

Os impactos da divulgação dos eventos da Zelotes no valor das ações do Santander, Gerdau e Bradesco, foram analisados através do modelo de estudo de eventos apresentado por Campbell, Lo e Mackinlay (1997). Assim, os procedimentos adotados para o estudo foram:
- Definição do retorno diário das ações do Santander, Gerdau e Bradesco através do software "The Economatica System". Foram realizadas 120 observações de retornos diários das empresas e do IBOVESPA;

- Determinação do retorno do IBOVESPA durante o mesmo período através do mesmo software;

- Estimativa dos parâmetros de regressão linear das ações do Santander, Gerdau e Bradesco em relação ao mercado;

- Definição das janelas dos eventos, com a utilização de 3 dias antes e 3 depois em que os eventos da Zelotes fizeram referência às empresas analisadas.

- As janelas do Santander foram: $1^{\circ}$ evento foi em 23/06/20I5 ( $I^{a}$ janela foi de 18 a 22/06/20I5, considerando que 20 e $21 / 06 / 2015$ foi final de semana, e de 24 a 26/06/20I6); $2^{\circ}$ evento foi em 06/05/2016 ( $2^{\mathrm{a}}$ janela foi de 03 a 05/05/2016 e de 09 a $11 / 05 / 2016$, considerando que 07 e 08/05/2016 foi final de semana).

- As janelas da Gerdau foram: $1^{\circ}$ evento foi em $25 / 02 / 2016$ ( $I^{a}$ janela foi de 22 a 24/02/2016 e de 26/02/20016 a $01 / 03 / 2016$, considerando que 27 e 28/02/2016 foi final de semana); $2^{\circ}$ evento foi em 16/05/2016 ( $2^{\mathrm{a}}$ janela foi de II a 13/05/2016 e de 17 a 19/05/2016, considerando que 14 e 15/05/2016 foi final de semana); $3^{\circ}$ evento foi em 26/05/2016 ( $3^{\mathrm{a}}$ janela foi de 23 a 25/05/2016 e de $30 / 05 / 2016$ a $01 / 06 / 2016)$. Como no Brasil foi feriado no dia 26/05/2016 (Corpus Christi) foi considerado como data do evento o dia seguinte, ou seja, 27/05/2016; 
- As janelas do Bradesco foram: $1^{\circ}$ evento foi em 31/05/2016 (I ${ }^{a}$ janela foi nos dias 25, 27 e 30/05/2016, considerando que 26/05 foi feriado e 28 e 29/05 foi final de semana, e de $1^{\circ}$ a 03/06/2016); $2^{\circ}$ evento foi em 03/06/2016 ( $2^{\mathrm{a}}$ janela foi de 31/05 a 02/06/2016 e de 06 a 08/06/2016, considerando que 04 e 05/06/2016 foi final de semana).

- Cálculo da diferença entre o retorno verificado durante as janelas dos eventos e do retorno normal estimado pelo modelo de mercado;

- Os parâmetros para estimativa do CAPM foram os seguintes: retornos diários para o período de 120 dias anteriores à janela do evento; retorno de mercado $=$ retorno diário do IBOVESPA; risk free = taxa Selic diária; Beta diário a partir da regressão linear da ação em relação ao IBOVESPA;

- Determinação da significância estatística dos retornos anormais: aplicação do Teste "T" para determinação dos diversos níveis de significância dos retornos determinados nas janelas dos eventos.

Após a realização dos testes, procedeuse à apresentação dos resultados empíricos, suas interpretações e conclusões.

\section{RESULTADOS DO ESTUDO DOS EVENTOS DA ZELOTES}

Os resultados dos impactos dos eventos da Zelotes no retorno das ações do Santander, Gerdau e Bradesco serão apresentados a seguir.

Análise dos impactos dos eventos da Zelotes

Foram analisados os retornos das ações do Santander (SANB4), da Gerdau (GGBR4) e do Bradesco (BBDC4), negociadas na BOVESPA, e do IBOVESPA nas janelas dos eventos da Zelotes. $O$ estudo tomou como base as ações PN (código 4), pois são as ações que possuem mais liquidez no mercado, portanto com um processo de formação de preços mais eficiente, dada a alta liquidez.

A análise foi fundamentada na teoria de estudo de eventos através da aplicação de técnicas investigativas, determinando o quanto eventos específicos são capazes de impactar os retornos das ações, gerando retornos anormais, ou seja, sem que o evento tivesse ocorrido.

Análise dos impactos dos eventos da Zelotes no Santander

Para analisar os impactos dos eventos da Zelotes na precificação das ações do Santander, foram identificados os retornos das ações do banco negociadas na BOVESPA e o IBOVESPA nas janelas dos eventos da Zelotes.

Janela I do Santander: evento do dia 23/06/20I5

Em 23/06/20I5 o Santander foi relacionado oficialmente pela primeira vez com a Zelotes, com a convocação de um de seus executivos para prestar esclarecimentos à CPI do Senado.

Os índices de fechamento e retornos diários reais do IBOVESPA, os índices de fechamento, os retornos diários reais e os retornos normais e anormais do Santander nas janelas do evento I serão apresentados na figura $\mathrm{I}$.

Uma vez identificados os resultados apurados nas janelas do evento I (quando a CPI convocou, em 23/06/2015, um executivo do 
FIGURA 1 - Resultados das janelas do evento 1 da IBOVESPA e do Santander

\begin{tabular}{|c|c|c|c|c|c|c|}
\hline Data & $\begin{array}{c}\text { Fechamento } \\
\text { IBOVESPA }\end{array}$ & $\begin{array}{c}\text { Retorno Diário } \\
\text { Real IBOVESPA }\end{array}$ & $\begin{array}{c}\text { Fechamento } \\
\text { Santander }\end{array}$ & $\begin{array}{c}\text { Retorno Diário } \\
\text { Real Santander }\end{array}$ & $\begin{array}{c}\text { Retorno Normal } \\
\text { Santander }\end{array}$ & $\begin{array}{c}\text { Retorno } \\
\text { Anormal } \\
\text { Santander }\end{array}$ \\
\hline $18 / 06 / 15$ & 54.238 & $1,842152 \%$ & 6,08 & $0,000000 \%$ & $0,699717 \%$ & $-0,69971 \%$ \\
\hline $19 / 06 / 15$ & 53.749 & $-0,905671 \%$ & 6,02 & $-1,019674 \%$ & $-0,234555 \%$ & $-0,78511 \%$ \\
\hline $22 / 06 / 15$ & 53.863 & $0,211872 \%$ & 6,01 & $-0,146520 \%$ & $0,145415 \%$ & $-0,29193 \%$ \\
\hline $23 / 06 / 15$ & 53.772 & $-0,169090 \%$ & 5,99 & $-0,440853 \%$ & $0,015886 \%$ & $-0,45673 \%$ \\
\hline $24 / 06 / 15$ & 53.842 & $0,130095 \%$ & 6,03 & $0,733679 \%$ & $0,117610 \%$ & $0,616069 \%$ \\
\hline $25 / 06 / 15$ & 53.175 & $-1,246547 \%$ & 6,26 & $3,730705 \%$ & $-0,350455 \%$ & $4,081160 \%$ \\
\hline $26 / 06 / 15$ & 54.016 & $1,569194 \%$ & 6,64 & $5,880026 \%$ & $0,606910 \%$ & $5,273116 \%$ \\
\hline
\end{tabular}

Fonte: autores

Santander citado na Zelotes), apresentados no quadro I, percebeu-se que o retorno diário real da IBOVESPA foi de -0,169090\%, do Santander foi de $-0,440853 \%$, o retorno normal do Santander, estimativa através do CAPM, foi de $0,015886 \%$, gerando um retorno anormal do Santander de $-0,45673 \%$ $(-0,440853 \%-0,015886 \%=-0,45673 \%)$. Uma vez identificados os resultados dessa janela, procedeu-se a um teste estatístico paramétrico de análise de variância, sendo que os resultados alcançados estão apresentados nas tabelas I, 2, 3 e 4.

2 a 4 apresentam o resultado da ANOVA da janela do evento I.

Analisando os resultados estatísticos gerados a partir da análise do evento I, visando testar o grau de significância

TABELA 1 - Resultados estatísticos da regressão da janela do evento 1

\begin{tabular}{lrr}
\hline & Estatística de regressão \\
\hline R múltiplo & 0,166619907 \\
\hline R-Quadrado & 0,027762193 \\
\hline R-quadrado ajustado & 0,01952289 \\
\hline Erro padrão & 0,027757977 \\
\hline Observações & 120 \\
\hline
\end{tabular}

Fonte: autores

TABELA 2 - Resultados a Anova da janela do evento 1

\begin{tabular}{l|c|c|c|c|c}
\hline & $G l$ & SQ & $M Q$ & $F$ & F de significação \\
\hline Regressão & 1 & 0,002596205 & 0,002596205 & 3,369483052 & 0,068932547 \\
\hline Resíduo & 118 & 0,090919625 & 0,000770505 & & \\
\hline Total & 119 & 0,093515829 & & & \\
\hline
\end{tabular}

Fonte: autores

TABELA 3 - Resultados a Anova da janela do evento 1

\begin{tabular}{l|c|c|c|c|c}
\hline & Coeficientes & Erro padrão & Stat $t$ & valor-P & 95\% inferiores \\
\hline Interseção & 0,000733771 & 0,002537667 & 0,289151759 & 0,772972641 & $-0,004291501$ \\
\hline Variável X1 & 0,340004569 & 0,185226498 & 1,83561517 & 0,068932547 & $-0,026794324$ \\
\hline
\end{tabular}

Fonte: autores

Tabela 4 - Resultados a Anova da janela do evento 1

\begin{tabular}{c|c|c|c}
\hline & $95 \%$ superiores & Inferior 95,0\% & Superior 95,0\% \\
\hline Interseção & 0,005759043 & $-0,004291501$ & 0,005759043 \\
\hline Variável X1 & 0,706803462 & $-0,026794324$ & 0,706803462 \\
\hline
\end{tabular}

Fonte: autores 
dos dados a partir do teste "T" para a análise da variância (ANOVA), é possível constatar que o $\mathrm{p}$-valor ficou inferior ao valor gerado pela Stat t, indicando que os resultando não são significantes, com 95\% de confiança, validando, portanto, a hipótese nula de que não há diferenças significativas entre o retorno normal e o retorno anormal.

Janela 2 do Santander: evento do dia 06/05/2016

No dia 06/05/2016 o Santander foi denunciado pelo MPF como participante do esquema, com uma fraude estimada de $R \$$ 3,34 bilhões. Nessa data, conforme figura 2, o retorno diário real da IBOVESPA foi de - I,690646\%, do Santander foi de - I,693852\%, o retorno normal do Santander, estimativa através do modelo de mercado CAPM, foi de $0,483766 \%$, gerando um retorno anormal do Santander de -2, I776I7\% (- I,693852\% $0,483766 \%=-2,|776| 7 \%)$.

Uma vez identificados os resultados dessa janela, procedeu-se a um teste estatístico paramétrico de análise de variância, sendo que os resultados alcançados estão apresentados nas tabelas 5, 6, 7 e 8.

FIGURA 2 - Resultados das janelas do evento 2 da IBOVESPA e do Santander

\begin{tabular}{|c|c|c|c|c|c|c|}
\hline Data & $\begin{array}{c}\text { Fechamento } \\
\text { IBOVESPA }\end{array}$ & $\begin{array}{c}\text { Retorno Diário } \\
\text { Real IBOVES- } \\
\text { PA }\end{array}$ & $\begin{array}{c}\text { Fechamento } \\
\text { Santander }\end{array}$ & $\begin{array}{c}\text { Retorno Diário } \\
\text { Real Santander }\end{array}$ & $\begin{array}{c}\text { Retorno Normal } \\
\text { Santander }\end{array}$ & $\begin{array}{c}\text { Retorno } \\
\text { Anormal } \\
\text { Santander }\end{array}$ \\
\hline $03 / 05 / 16$ & 53.561 & $-0,649480 \%$ & 7,75 & $-0,257732 \%$ & $0,350702 \%$ & $-0,608434 \%$ \\
\hline $04 / 05 / 16$ & 52.260 & $-2,458993 \%$ & 7,65 & $-1,298720 \%$ & $0,581963 \%$ & $-1,880682 \%$ \\
\hline $05 / 05 / 16$ & 52.552 & $0,557190 \%$ & 7,74 & $1,169604 \%$ & $0,196486 \%$ & $0,973118 \%$ \\
\hline $06 / 05 / 16$ & 51.671 & $-1,690646 \%$ & 7,61 & $-1,693852 \%$ & $0,483766 \%$ & $-2,177617 \%$ \\
\hline $09 / 05 / 16$ & 51.717 & $0,088985 \%$ & 7,62 & $0,131320 \%$ & $0,256324 \%$ & $-0,125004 \%$ \\
\hline $10 / 05 / 16$ & 50.990 & $-1,415701 \%$ & 7,80 & $2,334736 \%$ & $0,448627 \%$ & $1,886109 \%$ \\
\hline $11 / 05 / 16$ & 53.070 & $3,998226 \%$ & 7,70 & $-1,290340 \%$ & $-0,243287 \%$ & $-1,047053 \%$ \\
\hline
\end{tabular}

Fonte: autores

TABELA 5 - Resultados estatísticos da regressão da janela do evento 2

\begin{tabular}{l|r}
\hline \multicolumn{2}{c}{ Estatística de regressão } \\
\hline R múltiplo & 0,066303885 \\
\hline R-Quadrado & 0,004396205 \\
\hline R-quadrado ajustado & $-0,004041115$ \\
\hline Erro padrão & 0,038244299 \\
\hline Observações & 120 \\
\hline
\end{tabular}

Fonte: autores

TABELA 6 - Resultados a Anova da janela do evento 2

\begin{tabular}{l|c|c|c|c|c}
\hline & $g l$ & $S Q$ & $M Q$ & $F$ & F de significação \\
\hline Regressão & 1 & 0,000762091 & 0,000762091 & 0,521042827 & 0,471825139 \\
\hline Resíduo & 118 & 0,172589914 & 0,001462626 & & \\
\hline Total & 119 & 0,173352005 & & & \\
\hline
\end{tabular}

Fonte: autores

TABELA 7 - Resultados a Anova da janela do evento 2

\begin{tabular}{l|c|c|c|c|c}
\hline & Coeficientes & Erro padrão & Stat $t$ & valor-P & 95\% inferiores \\
\hline Interseção & 0,002676967 & 0,003499874 & 0,764875191 & 0,445872866 & $-0,004253736$ \\
\hline Variável X1 & $-0,127802676$ & 0,177052978 & $-0,721832964$ & 0,471825139 & $-0,478415774$ \\
\hline
\end{tabular}

Fonte: autores 
TABELA 8 - Resultados a Anova da janela do evento 2

\begin{tabular}{c|c|c|c}
\hline & 95\% superiores & Inferior 95,0\% & Superior 95,0\% \\
\hline Interseção & 0,009607669 & $-0,004253736$ & 0,009607669 \\
\hline Variável X1 & 0,222810423 & $-0,478415774$ & 0,222810423 \\
\hline
\end{tabular}

Fonte: autores

Analisando os resultados estatísticos gerados pelo evento 2 , através do teste "T" para a análise da variância (ANOVA), constatou-se que o $\mathrm{p}$-valor ficou inferior ao valor gerado pela Stat $t$, indicando que os resultando não são significantes, com $95 \%$ de confiança, validando, portanto, a hipótese nula, de que, assim como no evento I, também não há diferenças significativas entre o retorno normal e o retorno anormal.

Análise dos impactos dos eventos da Zelotes na Gerdau

Para analisar os impactos dos eventos da Zelotes na precificação das ações da Gerdau, foram identificados os retornos das ações da empresa negociadas na BOVESPA e o índice da IBOVESPA nas janelas dos eventos da Zelotes.
Janela I da Gerdau: evento do dia $25 / 02 / 2016$

No dia 25/02/2016 a Gerdau foi citada na Zelotes e passou a ser investigada por uma suposta compra de decisões no Carf que pode atingir o total de $R \$ 1,22$ bilhão.

Conforme figura 3 , o retorno diário real do IBOVESPA foi de -0,46921\% e da Gerdau foi de $-4,8922799 \%$, o retorno normal da Gerdau, estimado através do CAPM, foi de $-0,654164 \%$, portanto, o retorno anormal da Gerdau foi de $-4,238116 \%(-4,238116 \%-0,654164 \%$ $=-4,8922799 \%)$.

Com a identificação dos resultados do evento I da Gerdau, foram analisados estatisticamente os índices, sendo que os resultados alcançados serão apresentados nas tabelas 9 a 12 .

FIGURA 3 - Resultados das janelas do evento 1 da IBOVESPA e da Gerdau

\begin{tabular}{|c|c|c|c|c|c|c|}
\hline Data & $\begin{array}{c}\text { Fechamento } \\
\text { IBOVESPA }\end{array}$ & $\begin{array}{c}\text { Retorno Diário } \\
\text { Real } \\
\text { IBOVESPA }\end{array}$ & $\begin{array}{c}\text { Fechamento } \\
\text { Gerdau }\end{array}$ & $\begin{array}{c}\text { Retorno Diário } \\
\text { Real Gerdau }\end{array}$ & $\begin{array}{c}\text { Retorno Normal } \\
\text { Gerdau }\end{array}$ & $\begin{array}{c}\text { Retorno } \\
\text { Anormal Gerdau }\end{array}$ \\
\hline $22 / 02 / 16$ & 43.234 & $3,98982 \%$ & 4,13 & $0,9732437 \%$ & $4,748043 \%$ & $-3,774799 \%$ \\
\hline $23 / 02 / 16$ & 42.520 & $-1,66527 \%$ & 3,89 & $-5,9868249 \%$ & $-2,103211 \%$ & $-3,883614 \%$ \\
\hline $24 / 02 / 16$ & 42.084 & $-1,03069 \%$ & 3,77 & $-3,1334156 \%$ & $-1,334412 \%$ & $-1,799004 \%$ \\
\hline $25 / 02 / 16$ & 41.887 & $-0,46921 \%$ & 3,59 & $-4,8922799 \%$ & $-0,654164 \%$ & $-4,238116 \%$ \\
\hline $26 / 02 / 16$ & 41.593 & $-0,70436 \%$ & 3,49 & $-2,8250466 \%$ & $-0,939056 \%$ & $-1,885990 \%$ \\
\hline $29 / 02 / 16$ & 42.793 & $2,84427 \%$ & 3,53 & $1,1396135 \%$ & $3,360181 \%$ & $-2,220568 \%$ \\
\hline $01 / 03 / 16$ & 44.121 & $3,05613 \%$ & 3,60 & $1,9635975 \%$ & $3,616862 \%$ & $-1,653265 \%$ \\
\hline
\end{tabular}

Fonte: autores

TABELA 9 - Resultados estatísticos da regressão da janela do evento 1

\begin{tabular}{l|c}
\hline \multicolumn{2}{c}{ Estatística de regressão } \\
\hline R múltiplo & 0,539208801 \\
\hline R-Quadrado & 0,290746131 \\
\hline R-quadrado ajustado & 0,284735505 \\
\hline Erro padrão & 0,033653942 \\
\hline Observações & 120 \\
\hline
\end{tabular}

Fonte: autores 
TABELA 10 - Resultados a Anova da janela do evento 1

\begin{tabular}{l|c|c|c|c|c}
\hline & $g l$ & $S Q$ & $M Q$ & $F$ & F de significação \\
\hline Regressão & 1 & 0,054785562 & 0,054785562 & 48,37202148 & $2,09989 \mathrm{E}-10$ \\
\hline Resíduo & 118 & 0,133645363 & 0,001132588 & & \\
\hline Total & 119 & 0,188430925 & & & \\
\hline
\end{tabular}

Fonte: autores

TABELA 11 - Resultados a Anova da janela do evento 1

\begin{tabular}{l|c|c|c|c|c}
\hline & Coeficientes & Erro padrão & Stat $t$ & valor-P & $95 \%$ inferiores \\
\hline Interseção & $-0,000857055$ & 0,003076776 & $-0,27855635$ & 0,78107287 & $-0,006949909$ \\
\hline Variável X1 & 1,211520786 & 0,174194224 & 6,954999747 & $2,0998 \mathrm{E}-10$ & 0,866568798 \\
\hline
\end{tabular}

Fonte: autores

TABELA 12 - Resultados a Anova da janela do evento 1

\begin{tabular}{c|c|c|c}
\hline & $95 \%$ superiores & Inferior 95,0\% & Superior 95,0\% \\
\hline Interseção & 0,005235798 & $-0,006949909$ & 0,005235798 \\
\hline Variável X1 & 1,556472774 & 0,866568798 & 1,556472774 \\
\hline
\end{tabular}

Fonte: autores

Com a análise dos resultados estatísticos gerados no evento I, testando o grau de significância dos dados a partir do teste "T" para a análise da variância (ANOVA), foi possível constatar que o $p$-valor ficou inferior ao valor gerado pela Stat $t$, indicando que os resultando não são significantes, com 95\% de confiança, validando, portanto, a hipótese nula, de que não há diferenças significativas entre o retorno normal e o retorno anormal.

Janela 2 da Gerdau: evento do dia 16/05/2016

O evento da janela 2 ocorreu em
16/05/20 16, quando a PF indiciou o presidente do Grupo Gerdau pelos crimes de corrupção ativa, corrupção passiva, lavagem de dinheiro, tráfico de influência, entre outros.A figura 4 os resultados da janela 2 da Gerdau.

O resultado anormal da janela 2 da Gerdau de $-6,937435 \%$, foi apurado a partir da identificação do retorno real na data do evento $(-6,899287$ I\%) em comparação com o retorno normal estimado através do CAPM (0,038I48\%). Os valores apurados na figura 4 , foram analisados estatisticamente e os índices e os resultados alcançados serão apresentados nas tabelas $13,14,15$ e 16.

FIGURA 4 - Resultados das janelas do evento 2 da IBOVESPA e da Gerdau

\begin{tabular}{|c|c|c|c|c|c|c|}
\hline Data & $\begin{array}{c}\text { Fechamento } \\
\text { IBOVESPA }\end{array}$ & $\begin{array}{c}\text { Retorno Diário } \\
\text { Real IBOVES- } \\
\text { PA }\end{array}$ & $\begin{array}{c}\text { Fechamento } \\
\text { Gerdau }\end{array}$ & $\begin{array}{c}\text { Retorno Diário } \\
\text { Real Gerdau }\end{array}$ & $\begin{array}{c}\text { Retorno Normal } \\
\text { Gerdau }\end{array}$ & $\begin{array}{c}\text { Retorno } \\
\text { Anormal Gerdau }\end{array}$ \\
\hline $11 / 05 / 16$ & 52.764 & $-0,57827 \%$ & 7,33 & $1,5120563 \%$ & $-0,947031 \%$ & $2,459087 \%$ \\
\hline $12 / 05 / 16$ & 53.241 & $0,89996 \%$ & 7,05 & $-3,8947899 \%$ & $1,588324 \%$ & $-5,483114 \%$ \\
\hline $13 / 05 / 16$ & 51.804 & $-2,73614 \%$ & 6,60 & $-6,5957968 \%$ & $-4,648068 \%$ & $-1,947729 \%$ \\
\hline $16 / 05 / 16$ & 51.802 & $-0,00386 \%$ & 6,16 & $-6,8992871 \%$ & $0,038148 \%$ & $-6,937435 \%$ \\
\hline $17 / 05 / 16$ & 50.839 & $-1,87650 \%$ & 6,00 & $-2,6317308 \%$ & $-3,173669 \%$ & $0,541938 \%$ \\
\hline $18 / 05 / 16$ & 50.561 & $-0,54832 \%$ & 5,91 & $-1,5113638 \%$ & $-0,895679 \%$ & $-0,615685 \%$ \\
\hline $19 / 05 / 16$ & 50.132 & $-0,85210 \%$ & 5,67 & $-4,1456714 \%$ & $-1,416693 \%$ & $-2,728979 \%$ \\
\hline
\end{tabular}

Fonte: autores 
TABELA 13 - Resultados estatísticos da regressão da janela do evento 2

\begin{tabular}{l|c}
\hline \multicolumn{2}{c}{ Estatística de regressão } \\
\hline R múltiplo & 0,659968626 \\
\hline R-Quadrado & 0,435558587 \\
\hline R-quadrado ajustado & 0,430775185 \\
\hline Erro padrão & 0,038074764 \\
\hline Observações & 120 \\
\hline
\end{tabular}

Fonte: autores

TABELA 14 - Resultados a Anova da janela do evento 2

\begin{tabular}{l|c|c|c|c|c}
\hline & $g l$ & $S Q$ & $M Q$ & $F$ & F de significação \\
\hline Regressão & 1 & 0,13200311 & 0,13200311 & 91,05624078 & $2,43499 \mathrm{E}-16$ \\
\hline Resíduo & 118 & 0,171063146 & 0,001449688 & & \\
\hline Total & 119 & 0,303066256 & & & \\
\hline
\end{tabular}

Fonte: autores

TABELA 15 - Resultados a Anova da janela do evento 2

\begin{tabular}{l|c|c|c|c|c}
\hline & Coeficientes & Erro padrão & Stat $t$ & valor-P & $95 \%$ inferiores \\
\hline Interseção & 0,000447697 & 0,003478882 & 0,12868996 & 0,897822063 & $-0,006441437$ \\
\hline Variável X1 & 1,715130042 & 0,179738948 & 9,542339377 & $2,43499 \mathrm{E}-16$ & 1,359197991 \\
\hline
\end{tabular}

Fonte: autores

TABELA 16 - Resultados a Anova da janela do evento 2

\begin{tabular}{c|c|c|c}
\hline & 95\% superiores & Inferior 95,0\% & Superior 95,0\% \\
\hline Interseção & 0,007336831 & $-0,006441437$ & 0,007336831 \\
\hline Variável X1 & 2,071062093 & 1,359197991 & 2,071062093 \\
\hline
\end{tabular}

Fonte: autores

Analisando os resultados estatísticos gerados pelo evento 2, através do teste " $T$ " para a análise da variância (ANOVA), é possível constatar que o $\mathrm{p}$-valor ficou inferior ao valor gerado pela Stat $t$, indicando que os resultando não são significantes, com 95\% de confiança, validando, portanto, a hipótese nula, de que, assim como no evento I, também não há diferenças significativas entre o retorno normal e o retorno anormal.

Janela 3 da Gerdau: evento

do dia 26/05/2016

No dia 26/05/2016 ocorreu o evento da janela 3, quando foi protocolada em um tribunal de Nova York uma ação coletiva de investidores que compraram ações da Gerdau entre 02/06/20 I I e 15/05/20 I6. A figura 5 apresenta o resultado anormal da Ger- dau apurado no dia 27/05 de -0,922866\% $(-0,922866 \%-I, 507809 \%=-2,4306752 \%)$. Foi utilizado o dia seguinte ao evento, ou seja, 27, pois dia 26 foi feriado nacional.

Como nos eventos anteriores, procedeu-se a um teste estatístico paramétrico de análise de variância, sendo que os resultados alcançados estão apresentados nas tabelas 9 a 12.

A análise estatística dos índices e os resultados do evento 3 da Gerdau serão apresentados nas tabelas 17 a 20.

As tabelas 18 a 20 apresentam o resultado da ANOVA da janela do evento 3.

Seguindo os mesmos critérios realizados para o evento I e evento 2 , a análise estatística realizada para o evento 3 , através do teste "T" para a análise da variância (ANOVA), é possível constatar que o p-va- 
FIGURA 5 - Resultados das janelas do evento 3 da IBOVESPA e da Gerdau

\begin{tabular}{|c|c|c|c|c|c|c|}
\hline Data & $\begin{array}{c}\text { Fechamento } \\
\text { IBOVESPA }\end{array}$ & $\begin{array}{c}\text { Retorno Diário } \\
\text { Real IBOVES- } \\
\text { PA }\end{array}$ & $\begin{array}{c}\text { Fechamento } \\
\text { Gerdau }\end{array}$ & $\begin{array}{c}\text { Retorno Diário } \\
\text { Real Gerdau }\end{array}$ & $\begin{array}{c}\text { Retorno Normal } \\
\text { Gerdau }\end{array}$ & $\begin{array}{c}\text { Retorno } \\
\text { Anormal Gerdau }\end{array}$ \\
\hline $23 / 05 / 16$ & 49.330 & $-0,79151 \%$ & 5,88 & $1,1976191 \%$ & $-1,367650 \%$ & $2,565269 \%$ \\
\hline $24 / 05 / 16$ & 49.345 & $0,03040 \%$ & 5,69 & $-3,2846514 \%$ & $0,014758 \%$ & $-3,299409 \%$ \\
\hline $25 / 05 / 16$ & 49.482 & $0,27725 \%$ & 5,83 & $2,4306752 \%$ & $0,429945 \%$ & $2,000731 \%$ \\
\hline $27 / 05 / 16$ & 49.051 & $-0,87484 \%$ & 5,69 & $-2,4306752 \%$ & $-1,507809 \%$ & $-0,922866 \%$ \\
\hline $30 / 05 / 16$ & 48.964 & $-0,17752 \%$ & 5,60 & $-1,5943650 \%$ & $-0,334964 \%$ & $-1,259401 \%$ \\
\hline $31 / 05 / 16$ & 48.471 & $-1,01197 \%$ & 5,57 & $-0,5371544 \%$ & $-1,738448 \%$ & $1,201293 \%$ \\
\hline $01 / 06 / 16$ & 49.012 & $1,10995 \%$ & 5,64 & $1,2489012 \%$ & $1,830493 \%$ & $-0,581592 \%$ \\
\hline
\end{tabular}

Fonte: autores

TABELA 17 - Resultados estatísticos da regressão da janela do evento 3

\begin{tabular}{l|c}
\hline \multicolumn{2}{c}{ Estatística de regressão } \\
\hline R múltiplo & 0,662090035 \\
\hline R-Quadrado & 0,438363215 \\
\hline R-quadrado ajustado & 0,433603581 \\
\hline Erro padrão & 0,038059842 \\
\hline Observações & 120 \\
\hline
\end{tabular}

Fonte: autores

TABELA 18 - Resultados a Anova da janela do evento 3

\begin{tabular}{l|c|c|c|c|c}
\hline & $g l$ & $S Q$ & $M Q$ & $F$ & F de significação \\
\hline Regressão & 1 & 0,13341189 & 0,13341189 & 92,10019838 & $1,80933 \mathrm{E}-16$ \\
\hline Resíduo & 118 & 0,170929089 & 0,001448552 & & \\
\hline Total & 119 & 0,304340979 & & & \\
\hline
\end{tabular}

Fonte: autores

TABELA 19 - Resultados a Anova da janela do evento 3

\begin{tabular}{l|c|c|c|c|c}
\hline & Coeficientes & Erro padrão & Stat $t$ & valor- $P$ & 95\% inferiores \\
\hline Interseção & $-0,000363783$ & 0,003474689 & $-0,10469525$ & 0,91679538 & $-0,007244614$ \\
\hline Variável X1 & 1,681944155 & 0,175259387 & 9,596884827 & $1,80933 \mathrm{E}-16$ & 1,334882854 \\
\hline
\end{tabular}

Fonte: autores

TABELA 20 - Resultados a Anova da janela do evento 3

\begin{tabular}{c|c|c|c}
\hline & 95\% superiores & Inferior 95,0\% & Superior 95,0\% \\
\hline Interseção & 0,006517047 & $-0,007244614$ & 0,006517047 \\
\hline Variável X1 & 2,029005456 & 1,334882854 & 2,029005456 \\
\hline
\end{tabular}

Fonte: autores

lor ficou inferior ao valor gerado pela Stat t, indicando que os resultando não são significantes, com $95 \%$ de confiança, validando, portanto, a hipótese nula de que não há diferenças significativas entre o retorno normal e o retorno anormal.

Análise dos impactos dos eventos da Zelotes no Bradesco
A análise dos impactos dos 2 eventos da Zelotes na precificação das ações do Bradesco, serão apresentados a seguir.

Janela I do Bradesco: evento do dia $31 / 05 / 2016$

Em 3I de maio de 2016 a PF indiciou o presidente do Bradesco como participante do esquema de compra de decisões no Carf, 
com uma fraude estimada de $R \$ 2,75$ bilhões.

O retorno anormal do evento I do Bradesco foi de $-3,97336 \%$, apurado pela diferença entre o retorno diário real de $-5,1293294 \%$ em comparação com o re- torno normal do banco de $-1,155965 \%$, conforme figura 6 .

A análise estatística dos índices e os resultados do evento I do Bradesco serão apresentados nas tabelas 21 a 24.

FIGURA 6 - Resultados das janelas do evento 1 da IBOVESPA e do Bradesco

\begin{tabular}{|c|c|c|c|c|c|c|}
\hline Data & $\begin{array}{c}\text { Fechamento } \\
\text { IBOVESPA }\end{array}$ & $\begin{array}{c}\text { Retorno Diário } \\
\text { Real IBOVESPA }\end{array}$ & $\begin{array}{c}\text { Fechamento } \\
\text { Bradesco }\end{array}$ & $\begin{array}{c}\text { Retorno Diário } \\
\text { Real Bradesco }\end{array}$ & $\begin{array}{c}\text { Retorno Normal } \\
\text { Bradesco }\end{array}$ & $\begin{array}{c}\text { Retorno } \\
\text { Anormal } \\
\text { Bradescor }\end{array}$ \\
\hline $25 / 05 / 16$ & 49.482 & $0,27725 \%$ & 24,21 & $-0,4086642 \%$ & $0,521828 \%$ & $-0,93049 \%$ \\
\hline $27 / 05 / 16$ & 49.051 & $-0,87484 \%$ & 23,87 & $-1,4436217 \%$ & $-0,977509 \%$ & $-0,46611 \%$ \\
\hline $30 / 05 / 16$ & 48.964 & $-0,17752 \%$ & 23,80 & $-0,2912421 \%$ & $-0,070020 \%$ & $-0,22122 \%$ \\
\hline $31 / 05 / 16$ & 48.471 & $-1,01197 \%$ & 22,61 & $-5,1293294 \%$ & $-1,155965 \%$ & $-3,97336 \%$ \\
\hline $01 / 06 / 16$ & 49.012 & $1,10995 \%$ & 23,02 & $1,8253440 \%$ & $1,605502 \%$ & $0,219842 \%$ \\
\hline $02 / 06 / 16$ & 49.887 & $1,76953 \%$ & 23,77 & $3,1771857 \%$ & $2,463881 \%$ & $0,713305 \%$ \\
\hline $03 / 06 / 16$ & 50.619 & $1,45666 \%$ & 23,89 & $0,4997928 \%$ & $2,015722 \%$ & $-1,51592 \%$ \\
\hline
\end{tabular}

Fonte: autores

TABELA 21 - Resultados estatísticos da regressão da janela do evento 1

\begin{tabular}{l|c}
\hline \multicolumn{2}{c}{ Estatística de regressão } \\
\hline R múltiplo & 0,875159289 \\
\hline R-Quadrado & 0,76590378 \\
\hline R-quadrado ajustado & 0,763919914 \\
\hline Erro padrão & 0,014253819 \\
\hline Observações & 120 \\
\hline
\end{tabular}

Fonte: autores

TABELA 22 - Resultados a Anova da janela do evento 1

\begin{tabular}{l|c|c|c|c|c}
\hline & $g l$ & $S Q$ & $M Q$ & $F$ & $F$ de significação \\
\hline Regressão & 1 & 0,078437599 & 0,078437599 & 386,0662347 & $5,2019 \mathrm{E}-39$ \\
\hline Resíduo & 118 & 0,02397422 & 0,000203171 & & \\
\hline Total & 119 & 0,102411818 & & & \\
\hline
\end{tabular}

Fonte: autores

TABELA 23 - Resultados a Anova da janela do evento 1

\begin{tabular}{l|c|c|c|c|c}
\hline & Coeficientes & Erro padrão & Stat $t$ & valor-P & 95\% inferiores \\
\hline Interseção & 0,001610107 & 0,001301501 & 1,237115522 & 0,218500509 & $-0,000967219$ \\
\hline Variável X1 & 1,30140368 & 0,066234021 & 19,64856826 & $5,2019 \mathrm{E}-39$ & 1,170242286 \\
\hline
\end{tabular}

Fonte: autores

TABELA 24 - Resultados a Anova da janela do evento 1

\begin{tabular}{c|c|c|c}
\hline & $95 \%$ superiores & Inferior 95,0\% & Superior 95,0\% \\
\hline Interseção & 0,004187432 & $-0,000967219$ & 0,004187432 \\
\hline Variável X1 & 1,432565073 & 1,170242286 & 1,432565073 \\
\hline
\end{tabular}

Fonte: autores

Analisando os resultados estatísticos gerados a partir da análise do evento I, visando testar o grau de significância dos dados a partir do teste "T" para a análise da variância (ANOVA), é possível constatar que o $\mathrm{p}$-valor ficou inferior ao 
valor gerado pela Stat $t$, indicando que os resultando não são significantes, com $95 \%$ de confiança. Esse resultado valida a hipótese nula de que não há diferença significativa entre o retorno normal e o retorno anormal.

Janela 2 do Bradesco: evento do dia 03/06/2016

Assim como aconteceu com a Gerdau, em 03/06/2016 foi aberto um processo contra o Bradesco em um tribunal de Manhattan em nome de investidores que adquiriram ADRs do Bradesco entre 30/04/20I2 e 3I/05/20I6.

Os retornos do IBOVESPA e do Bradesco nas janelas do evento 2 serão apresentados na figura 7.

O retorno anormal do Santander nessa janela foi de -I,5I5929\% (2,0I5722\% de retorno normal e $0,4997928 \%$ de retorno real).

Após identificados os resultados apurados nas janelas do evento 2 , foram analisados estatisticamente e os índices e os resultados alcançados serão apresentados nas tabelas 25 a 28.

FIGURA 7 - Resultados das janelas do evento 2 da IBOVESPA e do Bradesco

\begin{tabular}{|c|c|c|c|c|c|c|}
\hline Data & $\begin{array}{c}\text { Fechamento } \\
\text { IBOVESPA }\end{array}$ & $\begin{array}{c}\text { Retorno Diário } \\
\text { Real } \\
\text { IBOVESPA }\end{array}$ & $\begin{array}{c}\text { Fechamento } \\
\text { Santander }\end{array}$ & $\begin{array}{c}\text { Retorno Diário } \\
\text { Real Santander }\end{array}$ & $\begin{array}{c}\text { Retorno Normal } \\
\text { Santander }\end{array}$ & $\begin{array}{c}\text { Retorno Anormal } \\
\text { Santander }\end{array}$ \\
\hline $31 / 05 / 16$ & 48.471 & $-1,01197 \%$ & 22,61 & $-5,129329 \%$ & $-1,155965 \%$ & $-3,973365 \%$ \\
\hline $01 / 06 / 16$ & 49.012 & $1,10995 \%$ & 23,02 & $1,8253440 \%$ & $1,605502 \%$ & $0,219842 \%$ \\
\hline $02 / 06 / 16$ & 49.887 & $1,76953 \%$ & 23,77 & $3,1771857 \%$ & $2,463881 \%$ & $0,713305 \%$ \\
\hline $03 / 06 / 16$ & 50.619 & $1,45666 \%$ & 23,89 & $0,4997928 \%$ & $2,015722 \%$ & $-1,515929 \%$ \\
\hline $06 / 06 / 16$ & 50.431 & $-0,37209 \%$ & 23,75 & $-0,5833350 \%$ & $-0,375496 \%$ & $-0,207839 \%$ \\
\hline $07 / 06 / 16$ & 50.487 & $0,11098 \%$ & 24,05 & $1,2871259 \%$ & $0,256158 \%$ & $1,030968 \%$ \\
\hline $08 / 06 / 16$ & 51.629 & $2,23677 \%$ & 24,81 & $3,0871664 \%$ & $3,035771 \%$ & $0,051395 \%$ \\
\hline
\end{tabular}

Fonte: autores

TABELA 25 - Resultados estatísticos da regressão da janela do evento 2

\begin{tabular}{l|c}
\hline \multicolumn{2}{c}{ Estatística de regressão } \\
\hline R múltiplo & 0,867874864 \\
\hline R-Quadrado & 0,753206779 \\
\hline R-quadrado ajustado & 0,751115311 \\
\hline Erro padrão & 0,014684349 \\
\hline Observações & 120 \\
\hline
\end{tabular}

Fonte: autores

TABELA 26 - Resultados a Anova da janela do evento 2

\begin{tabular}{l|c|c|c|c|c}
\hline & $g l$ & $S Q$ & $M Q$ & $F$ & $F$ de significação \\
\hline Regressão & 1 & 0,077655531 & 0,077655531 & 360,1330678 & $1,18333 \mathrm{E}-37$ \\
\hline Resíduo & 118 & 0,025444352 & 0,00021563 & & \\
\hline Total & 119 & 0,103099883 & & & \\
\hline
\end{tabular}

Fonte: autores

TABELA 27 - Resultados a Anova da janela do evento 2

\begin{tabular}{l|c|c|c|c|c}
\hline & Coeficientes & Erro padrão & Stat $t$ & valor- $P$ & 95\% inferiores \\
\hline Interseção & 0,001110421 & 0,001341152 & 0,827960498 & 0,409364283 & $-0,001545425$ \\
\hline Variável X 1 & 1,307570851 & 0,068902302 & 18,97717228 & $1,18333 \mathrm{E}-37$ & 1,171125535 \\
\hline
\end{tabular}

Fonte: autores 
TABELA 28 - Resultados a Anova da janela do evento 2

\begin{tabular}{c|c|c|c}
\hline & 95\% superiores & Inferior 95,0\% & Superior 95,0\% \\
\hline Interseção & 0,003766266 & $-0,001545425$ & 0,003766266 \\
\hline Variável X1 & 1,444016167 & 1,171125535 & 1,444016167 \\
\hline
\end{tabular}

Fonte: autores

A análise dos resultados estatísticos gerados pelo evento 2 , através do teste " $T$ " para a análise da variância (ANOVA), permite constatar que o $p$-valor ficou inferior ao valor gerado pela Stat $t$, indicando que os resultando não são significantes, com 95\% de confiança, validando, portanto, a hipótese nula.

A validação da hipótese nula em todas as janelas dos eventos da Zelotes que envolveu as três empresas analisadas, indicam que não existem diferenças significativas entre os retornos normais e anormais nos preços de negociação das ações, caracterizando, portanto, que esses eventos da Zelotes não afetaram o mercado acionário do Santander, da Gerdau e do Bradesco, indicando que o mercado não reage de maneira significativa ao anúncio dos eventos.

\section{CONSIDERAÇÕES FINAIS}

Os últimos acontecimentos vivenciados no Brasil, incluindo o impedimento do Presidente eleito em 2014, tem aberto uma ampla discussão na sociedade sobre as relações entre os setores público privado, buscando aumentar a transparência na gestão das coisas públicas. A aumento do número de agentes públicos punidos nas operações do MPF e PF, tem procurado coibir o uso distorcido dos recursos públicos, que poderá fortalecer as instituições e aumentar a qualidade do gasto público no médio e longo prazo.

Porém, mesmo que esses movimentos estejam alterando as atuais condições políticas e econômicas do país, depre- ende-se do resultado alcançado na pesquisa que a cultura da corrupção já faz parte do processo negocial brasileiro. Essa constatação pode ser comprovada com a análise dos resultados do estudo dos eventos da Zelotes, onde grandes empresas que negociam suas ações na BOVESPA, mesmo que estejam sendo investigadas pelo MPF e pela $P F$, não houve impacto nos valores de negociação de suas ações quando da externalização dos eventos em que foram citadas, indicando que os resultando apurados nos estudos não são significantes, com $95 \%$ de confiança, validando, portanto, a hipótese nula, ou seja, não há diferenças significativas entre o retorno normal e o retorno anormal.

Todos eventos analisados nesse estudo foram obtidos de informações públicas divulgadas pelo MPF e pela PF, publicadas nos principais jornais brasileiros. Muitos processos das 70 empresas investigadas por suspeitas de manipulação, entre 2005 e 2013, de decisões do Carf, ainda estão em fase de instrução e julgamento. $O$ foco desse estudo não foram as empresas envolvidas, mas a divulgação dos eventos pelo MPF e PF e seus impactos nos preços das ações da empresas envolvidas.

Após esse estudo constatou-se, portanto, que o mercado de capitais brasileiro, pelo menos no que diz respeito a essas ocorrências e para essas empresas analisadas, incorporou na precificação das ações a ocorrência sistêmica de corrupção, consi- 
derando que não houve afetação de forma significativa e consistente o desempenho das ações, verificado com os testes estatísticos, não dando resultados significantes.

Assim, analisando os resultados alcançados no estudo, foi possível concluir, preliminarmente, que o mercado foi indiferente, no longo prazo, às denúncias envolvendo as empesas analisadas, corroborando a tese de que o mercado brasileiro não reage de maneira significativa aos problemas de corrupção.

Em estudos futuros recomenda-se a ampliação do número de empresas citadas em outras Operações do MPF e da PF, relacionadas com corrupção de empresas que tenham suas ações negociadas na BOVESPA, buscando avigorar constatação de que o mercado de capitais brasileiro precifica a corrupção em suas negociações. 


\section{REFERÊNCIAS}

ARTHUR, M. M. Share price reactions to work-family initiatives: An institutional perspective. Academy of Management Journal, v.46, n.4, p.497-505, 2003.

ASHLEY, J.W. Stock Prices and Changes in Earnings and Dividends. Journal of Political Economy, v.70, n. I, p.82-85, 1962.

BALL, R.; BROWN, P. An empirical evaluation of accounting income numbers. Journal of accounting research, p. I59-178, 1968.

BALTAR, P.A. Estagnação da economia, abertura e crise do emprego urbano no Brasil. Economia e Sociedade, v.5, n. I, p.75-I I I, 2016.

BAQUERO, M. Corrupção, cultura política e capital social negativo no Brasil. Revista Debates, v.9, n.2, p.I39-I57, 2015.

BARKER, C.A. Effective Stock Splits. Harvard Business Review, v.34, n. I, p.I0I-106, 1956. Stock Splits in a Bull Market. Harvard Business Review, v.35, n.3, p.72-79, 1957.

Evaluation of Stock Dividends. Harvard Business Review, v.36, n.4, p.99-I |4, 1958.

BATISTELLA, F. D. et al. Retornos de ações e governança corporativa: um estudo de eventos. In: Congresso USP de controladoria e contabilidade. Universidade de São Paulo, São Paulo, 2004.

BERGMANN, R. et al. Avaliação dos Processos de Fusões e Aquisições no setor Bancário Brasileiro por meio de Estudo de Eventos. Revista Brasileira de Gestão de Negócios, v.17, n.56, p.II05I। I5, 2015.

BROWN, S. J.;WARNER, J. B. Measuring security price performance. Journal of Financial Economics, Amsterdam: North Holland, v.8, n.3, p.205-258, Sept. 1980.
BROWN, S. J.; WARNER, J. B. Using daily Stock Returns. Journal of Financial Economics, Amsterdam: North Holland, v. I4, n. I, p. 3-3I, Mar. 1985.

CACCIAMALI, M. C. Globalização e processo de informalidade. Economia e sociedade, v.9, n.l, p. I53-I74, 2016.

CAMARGOS, M.A.; BARBOSA, F.V. Estudos de evento: teoria e operacionalização. Caderno de Pesquisas em Administração, São Paulo, v. I0, n.3, p.0I-20, jul/set 2003.

CAMPBELL, J.Y.; LO, A.W.; MACKINLAY,A. C. The econometrics of financial

markets. New Jersey: Princeton University Press, 1997.

CAVALCANTE, L. M. T. et al. Sustentabilidade empresarial e valor das ações: uma análise na bolsa de valores de São Paulo. Revista de Gestão Social e Ambiental, v.3, n. I, p.70-86, 2009.

COHEN,A.;WANG, CY. How do staggered boards affect shareholder value? Evidence from a natural experiment. Journal of Financial Economics, v.lI0, n.3, p.627$641,2013$.

DEMIGUEL,V.; NOGALES, J.; UPPAL, R. Stock return serial dependence and out-of-sample portfolio performance. Review of Financial Studies, v.27, n.4, p. 1031 - I073, 2014.

DOLLEY, C. Characteristics and procedure of common stock split-ups. Harvard Business Review, v. I I, n.3, p.3 I6-326, 1933.

ELBANNAN, M.A. The Capital Asset Pricing Model: An Overview of the Theory. International Journal of Economics and Finance, v.7, n. I, 2015.

EXAME. Embraer, BRF e Petrobras saem do foco da Zelotes. Disponível em: http://exame.abril.com. $\mathrm{br} /$ negocios/noticias/embraer-brf-e-petrobras-saem-do-foco-dazelotes. Data acesso: I5/set/2016.

FAMA, E. F. et al. The adjustment of stock prices to new information. International economic review, v. I0, n. I, p. I-2I, 1969.

FOLHA DE SÃO PAULO. Entenda a Operação Zelotes da Polícia Federal. Disponível em: http:// wwwl folha.uol.com.br/mercado/20 I 5/04/ I 6 I I 246-entenda-a -operacao-zelotes-da-policia-federal.shtml. Data acesso: 12/set/2016.

GIL, A. C. Como elaborar projetos de pesquisa. São Paulo,Atlas, 2010. GONÇALVES, Rodrigo de Souza et al. Social disclosure and cost of equity in public companies in Brazil. Revista Contabilidade \& Finanças, v.24, n.62, p. I I3-124, 2013.

GRULLON, G.; MICHENAUD, S.;WESTON, P. The real effects of shortselling constraints. Review of Financial Studies, p. hhv0 I3, 2015.

IKENBERRY, D. L.; RAMNATH, S. Underreaction to self-selected news events: The case of stock splits. Review of Financial Studies, v. I5, n.2, p.489-526, 2002.

JESUS, D. V. Equilíbrio e oportunidade: Brasil e China frente à crise internacional (2007-2009)-DOI: I0.9732/P. 0034-7I91.2012 v104pl79. Revista Brasileira de Estudos Políticos, v. I04, p. I79-206, 2012.

JUNIOR, M. L.; CARVALHO, V. G. Impacto Ambiental e Retorno Acionário de Companhias Listadas na BM\&FBovespa. EmpíricaBR-Revista Brasileira de Gestão, Negócio e Tecnologia da Informação, v. I, n. I, p.43-54, 20 I 5. KAN, R.; ROBOTTI, C.; SHANKEN, J. Pricing Model Performance and the Two-Pass Cross-Sectional Regression Methodology. The Journal of Finance,v.68,n.6,p.26I7-2649, 2013. 
LINTNER, J. The valuation of risk assets and the selection of risky investments in stock portfolios and capital budgets. The review of economics and statistics, p. I3-37, 1965.

LUCCHESI, E. P.; FAMÁ, R. O impacto das decisões de investimento das empresas no valor de mercado das ações negociadas na Bovespa no período de 1996 a 2003. Revista de Administração, v.42, n.2, p.249-260, 2007.

MATIAS-PEREIRA, J.A economia brasileira frente à crise financeira e econômica mundial. Dissertação de mestrado, Universidade de Brasília, 2009.

MATIAS-PEREIRA, J. et al. Gestão das Políticas Fiscal e Monetária: Os efeitos colaterais da crise mundial no crescimento da economia brasileira. Revista Observatorio de la Economía Latinoamericana, v. I48, p. I-23, 20 I I.

MATTOSO, J.. Mudanças estruturais e trabalho no Brasil. Economia e Sociedade, v.7,n. I, p.2।3-243, 2016. MEDEIROS, O. R.; MATSUMOTO, AIberto Shigueru. Emissões públicas de ações, volatilidade e insider information na Bovespa. Revista contabilidade \& finanças, v. 17 , n.40, p.25-36, 2006.

MINISTÉRIO PÚBLICO FEDERAL. Atuação da MPF - Casos emblemáticos. Disponível em: http://www.combateacorrupcao. mpf.mp.br/atuacao-do-mpf. Data de acesso: 30/mai/2016.

MITCHELL, M. L.; STAFFORD, E. Managerial Decisions and Long-Term Stock Price Performance*. The Journal of Business, v.73, n.3, p.287-329, 2000.

MYERS, J. H.; BAKAY, Archie J. Influence of stock split-ups on market price. Harvard Business Review, v.26, n.2, p.25I-255, 1948.

NASCIMENTO, F. S. Muito além do moralismo: as causas da impro- bidade no Brasil. InterEspaço: Revista de Geografia e Interdisciplinaridade, v.l, n.2, p.169I87, 2015.

NASCIMENTO, C L.; BARACHO, H. U.. Corrupção e improbidades nas contratações públicas que prejudicam o desenvolvimento sustentável. Direito e Desenvolvimento, v.6, n. I2, p.39-6I, 2016. NERY, N.; GABRIEL, M. PF inclui Petrobras e partido PP em investigação sobre fraude fiscal. Disponível em: http://wwwl.folha. uol.com.br/mercado/20/5/03/ | 610770-pf-inclui-petrobras-e -partido-pp-em-investigacaosobre-fraude-fiscal.shtml. Data acesso: 08/jun/2016.

PAULA, L. R.;ALVES JR,A. J. External financial fragility and the 1998-1999 Brazilian currency crisis. Journal of Post Keynesian Economics, v.22, n.4, p.589-6I 7, 2000.

PAULO, E.; SARLO NETO, A.; SANTOS, M. C. Reação do preço das ações e intempestividade informacional do lucro contábil trimestral no Brasil. ASAA-Advances in Scientific and Applied Accounting, v.5, n. I, p.54-79, 2013.

PESSANHA, G. G. et al. Influências das fusões e aquisições no valor de mercado das instituições bancárias adquirentes. Contabilidade, Gestão e Governança, v.17, n.3, 2014.

PEREIRA, M. A.; SECURATO, J. R.; DE SOUSA,F. Efeito dos investimentos nos fundamentos e na reação de mercado de empresas brasileiras pré-operacionais e operacionais do período de 2006 a 2012. Revista de Administração, v.5I, n.I, p.56-7I, 20 I6.

PERESS, J.The media and the diffusion of information in financial markets: Evidence from newspaper strikes. The Journal of Finance, v.69, n.5, p.2007-2043, 2014.
POLINSKY, A. M.; SHAVELL, S. Handbook of Law and Economics. Vol. 2, New York, Elsevier, 2007.

QUEIROZ, J. W. Corruption-Can Brazil win this war? Master of Philosophy in System Dynamics. University of Bergen. 2015.

RICUPERO, R. et al. A crise internacional e seu impacto no Brasil. Estudos Avançados, v.22, n.64, p. | 85-2| 4, 2008.

ROSS, S. A. The arbitrage theory of capital asset pricing. Journal of economic theory, v.13, n.3, p.34l-360, 1976.

SAAD, C. Polícia Federal cumpre 33 mandados em nova fase da operação Zelotes. Disponível em: http://br.reuters.com/ article/domesticNews/idBRKCNOSKI5R20I5I026. Data de acesso: 30/mai/2016.

SHARPE, W. F. Capital asset prices: A theory of market equilibrium under conditions of risk. The journal of finance, v. 19, n.3, p.425-442, 1964.

Portfolio theory and capital markets. McGraw-Hill College, 1970.

SHARPE, W. F.; ALEXANDER, G. J.; BAILEY, J. Investments Prentice Hall. Englewood Cliffs, New Jersey, 1995.

TERRA, P. R. S.; LIMA, J. Governança corporativa e a reação do mercado de capitais à divulgação das informações contábeis. Revista contabilidade e finanças. São Paulo.v.4, n.42 p.35-49, set./dez. 2006.

ZABARANKIN, M; PAVLIKOV, K; URYASEV, S. Capital Asset Pricing Model (CAPM) with drawdown measure. European Journal of Operational Research, v.234, p.508-5I7, 2014.

WRIGHT, J. H. What does monetary policy do to long-term interest rates at the zero lower bound?. The Economic Journal, v.122, n.564, p.F447-F466, 2012. 